\title{
Entre relativisme et dogmatisme : la quête d'une troisième voie
}

L'enseignement de la pensée critique en France

Between relativism and dogmatism: The quest for a third way. Teaching critical thinking in France

Entre relativismo y dogmatismo: la búsqueda de una tercera vía. La enseñanza del pensamiento crítico en Francia

\section{Frank Smith}

\section{OpenEdition}

\section{Journals}

Édition électronique

URL : https://journals.openedition.org/ries/6137

DOI : 10.4000/ries.6137

ISSN : 2261-4265

\section{Éditeur}

France Education international

\section{Édition imprimée}

Date de publication : 30 avril 2018

Pagination : 71-81

ISBN : 978-2-85420-618-0

ISSN : $1254-4590$

Référence électronique

Frank Smith, "Entre relativisme et dogmatisme : la quête d'une troisième voie ", Revue internationale d'éducation de Sèvres [En ligne], 77 | avril 2018, mis en ligne le 30 avril 2020, consulté le 24 juin 2021. URL : http://journals.openedition.org/ries/6137 ; DOI : https://doi.org/10.4000/ries.6137 


\title{
Entre relativisme et dogmatisme : la quête d'une troisième voie
}

\section{L'enseignement de la pensée critique en France}

\author{
Frank Smith \\ Lycée Paul-Louis Courier et Université de Tours
}

Selon un sondage Ifop réalisé en décembre 2017, 9 \% des Français déclarent possible qu'en réalité « la Terre soit plate et non pas ronde comme on nous le dit depuis l'école », 16 \% pensent que les Américains ne sont jamais allés sur la Lune. Si seuls $3 \%$ affirment que les attentats de Charlie Hebdo et de l'Hyper Cacher de 2015 ont été fomentés par les services secrets, ils sont cependant $19 \%$ à estimer qu'il demeure des zones d'ombre (le pourcentage monte à $30 \%$ chez les 18-24 ans). Ces thèses conspirationnistes sont plus présentes chez les jeunes, témoignant de leur plus grande perméabilité à des thèses de ce type. Que faire, lorsque l'on est confronté à de tels propos à l'école ? Je proposerai d'abord un rapide aperçu de la situation en France, avant de proposer quelques éléments d'explication en me concentrant sur la situation d'un professeur de philosophie et de donner quelques pistes de solutions.

\section{UN BREF ÉTAT DES LIEUX}

Commençons par quelques considérations générales. En France, la situation est très contrastée : il n'y a guère de comparaison entre un grand lycée parisien et un établissement de banlieue défavorisée. Cette disparité géographique, qui reflète une réalité économique, se traduit en termes d'attitudes chez les élèves. La situation est contrastée aussi en fonction de la nature des établissements, qui relèvent de trois grandes catégories : les établissements d'enseignement public (plus de $80 \%$ des élèves), les établissements privés sous contrat (98\% des établissements privés) et les établissements privés hors contrat. Les établissements privés sous contrat sont tenus de respecter le même programme et leurs enseignants sont soumis aux mêmes modalités d'inspection et de recrutement que ceux du public. Si les établissements privés sont religieux, ce ne sont que secondairement des considérations confessionnelles qui sont à l'origine des choix entre public et privé sous contrat. De nombreux élèves - et quelques enseignants - font ainsi la navette entre les deux. Il en va autrement des établissements privés hors contrat qui ne sont pas astreints à suivre le même programme d'enseignement. Ajoutons une remarque : dans un livre stimulant, Jean-Paul Jouary (2002) observait que la France faisait preuve d'une grande complaisance 
envers les parasciences. Ainsi, il y aurait dans ce pays sensiblement autant de voyants que de médecins généralistes et les premiers généreraient un chiffre d'affaire supérieur aux seconds.

Dans le sondage de l'Ifop, on voit qu'il faut distinguer entre ceux qui nient expressément la « thèse officielle » et ceux qui en doutent. Il est évidemment très difficile de discuter avec ceux qui ne cherchent qu'à confirmer leurs a priori. Le principal enjeu est donc de ne pas laisser les "dubititationnistes » tomber dans le "négationnisme ». Il faut enfin noter que ces discours relèvent de cas différents : ils peuvent être le résultat d'une simple naïveté ou être le fruit d'une véritable manipulation; ils peuvent avoir des fondements religieux, politiques, etc., porter sur des savoirs scientifiques (théorie de l'évolution par exemple), des faits historiques (génocide des Juifs) ou sur des événements de la vie politique (attentats de 2015).

La première difficulté tient paradoxalement à l'accès facilité à l'information par le biais d'Internet, puisque celui-ci est devenu la première source d'information des jeunes français. Selon une enquête mondiale du Reuters Institute de 2016, 54 \% des Français de 18-24 ans citent en premier Internet comme source d'information ( $32 \%$ les sites web et $22 \%$ les réseaux sociaux) devant la télévision (27\%). Or le sociologue Gérald Bronner (2013) remarque une nette prédominance sur Internet des sites proposant des thèses contraires à l'orthodoxie scientifique, dans la mesure où les partisans de ces thèses sont très actifs. Sur chacun des sujets suivants : psychokinèse, monstre du Loch Ness, nocivité de l'aspartam, cercles de culture, astrologie, Bronner a consulté les trente premiers sites répertoriés par Google afin de voir s’ils étaient " favorables », " défavorables", «neutres » ou "sans objet » par rapport à ces sujets ( sans objet " signifiant que le site ne propose aucun point de vue concernant la croyance). Les résultats sont donnés dans le tableau suivant (les pourcentages ne portent que sur les sites prenant parti):

Tableau 1. Points de vue des 30 premiers sites répertoriés par Google sur 5 sujets

\begin{tabular}{|l|c|c|c|c|}
\hline & Favorables & Défavorables & Neutres & Sans objet \\
\hline Psychokinèse & $17(74 \%)$ & $6(26 \%)$ & 7 & 0 \\
\hline Monstre du Loch Ness & $14(78 \%)$ & $4(22 \%)$ & 4 & 8 \\
\hline Aspartam & $14(70 \%)$ & $6(30 \%)$ & 3 & 2 \\
\hline Cercles de culture & $14(87 \%)$ & $2(13 \%)$ & 12 & 1 \\
\hline Astrologie & $28(97 \%)$ & $1(3 \%)$ & 0 & 7 \\
\hline
\end{tabular}

Source : Frank Smith, d'après Bronner (2012).

L'offre est avant tout celle des «vérités alternatives ». Celui qui entreprend de toute bonne foi des recherches risque fort d'en venir à adhérer à ces thèses contraires aux théories scientifiques établies. Il est donc nécessaire 
d'apprendre aux élèves à maîtriser cet outil qu' est Internet. C'est notamment dans ce but qu'a été mise en place une éducation aux médias et à l'information dans la loi n 2013-595 du 8 juillet 2013 d'orientation et de programmation pour la refondation de l'école de la République. Au niveau du lycée, cette éducation ne fait pas l'objet de cours spécifique mais peut prendre place dans les «travaux personnels encadrés » (TPE) sous la direction des professeurs documentalistes ou faire l'objet d'un travail au sein de l'accompagnement personnalisé ou de l'enseignement moral et civique (EMC) souvent dispensé par les enseignants d'histoire-géographie. En définitive, elle est souvent très variable et, dans les faits, assez limitée.

Dans le domaine de la science, la principale remise en cause concerne le darwinisme. Plus marginalement, on entend des propos contestant le fait que l'homme ait marché sur la Lune, la rotondité de la Terre, le bienfait des vaccins ou le réchauffement climatique. Ce dernier point qui a pourtant fait l'objet d'un intense débat médiatique est peu contesté.

La remise en cause de la théorie de l'évolution par les élèves se fait sous couvert de religion. Cependant, le problème est en réalité politique puisqu'il s'agit de décider de ce qui nous gouverne : pouvoir laïque ou théocratie. Là encore, la situation dépend des publics auxquels on est confronté. Il n'y a pas de données chiffrées officielles mais il y a tout lieu de considérer que le créationnisme auquel la France doit faire face est majoritairement celui du fondamentalisme musulman, même si plusieurs témoignages laissent penser qu'il y a une recrudescence de ces propos chez les catholiques traditionnels et les quelques protestants français. Cependant il n'y a en France que peu d'attaques coordonnées. On a observé quelques actions comme l'envoi à plusieurs centaines d'établissements scolaires de l'Atlas de la création par le créationniste turc Harun Yahya en 2007. Malgré le légitime émoi qu'elles ont suscité, témoignant de la nécessité de rester vigilant ${ }^{1}$, ces actions demeurent marginales, même si, à l'époque, Georges Grousset, inspecteur académique régional en charge des sciences de la vie et de la terre s'en inquiétait :

il y a toujours eu des contestations, mais nous remarquons que de plus en plus de créationnistes cherchent à imposer leur avis dans les cours de biologie. ${ }^{2}$

La France est pourtant loin de connaître la situation d'autres pays comme les États-Unis, où, selon une enquête publiée par Science en 2011, 60 \% des professeurs de biologie enseignaient l'évolution au même titre que l'Intelligent Design ou encore l'Italie, où la ministre de l'instruction Letizia Moratti avait déposé, en 2004, un projet de loi pour supprimer la théorie de l'évolution des programmes secondaires. En France, de nombreuses actions sont menées (EMC, affichage en classe d'une charte de laïcité...) pour réaffirmer le caractère laïque de l'école publique.

1. Le ministère a rapidement réagi en organisant, dès novembre 2008 , un séminaire « Enseigner l'évolution » ouvert aux enseignants et inspecteurs de philosophie et de sciences naturelles.

2. Cité par Rachel Mulot, « Darwin et l'évolution contestés à l'école », Sciences et Avenir, février $2009, \mathrm{n}^{\circ} 744$. 
En histoire, les enseignants sont parfois confrontés au négationnisme. Or des personnes publiques, y compris des universitaires, ont, sous couvert de liberté d'expression et de recherche, défendu de telles thèses. Rappelons également qu'il $\mathrm{y}$ a eu quelques cas d'enseignants ayant tenu de tels propos. Outre la négation des faits, il s'agit pourtant d'un délit depuis la loi Gayssot du 13 juillet 1990 qui interdit la contestation de l'existence des crimes contre l'humanité. La difficulté particulière de ce discours tient à son statut ambivalent. Max Weber (1963) distinguait entre les faits qui relèvent de la science et les valeurs qui relèvent du politique. Mais le négationnisme n'est-il qu'un problème scientifique ? On peut en douter car derrière les affirmations supposées savantes, il y a une dénonciation haineuse. En fait la distinction wéberienne est inopérante, parce que les deux domaines idéalement séparés que sont la politique et la science sont en réalité intimement mêlés. La difficulté à laquelle est confronté l'enseignant est que ce soubassement idéologique est rarement assumé comme tel. Des élèves peuvent donc se réfugier derrière des propos qui manifestent leur antisémitisme sans l'exprimer. De tels propos doivent d'autant plus être condamnés qu'il est important pour la société de définir des assertions qui ne peuvent être remises en cause. Le négationnisme a des incidences politiques et sociales. La question à se poser est donc : quelles vérités la société peut-elle admettre de voir remises en cause sans risque pour elle et quelles remises en cause ne peut-elle tolérer?

\section{LES ORIGINES DE LA CONTESTATION : NAÏVETÉ, CROYANCES, MÉFIANCE, AFFIRMATION DE SOI}

Les deux cas évoqués ci-dessus sont le reflet d'idéologies délétères. Cependant, l'enseignant est aussi confronté à des difficultés qui relèvent de la naïveté épistémologique. Il ne s'agit pas tant d'obstacles épistémologiques au sens de Bachelard, c'est-à-dire de prétendues connaissances qui feraient obstacle au savoir, mais d'une conception erronée de la connaissance. On observe chez les élèves une grande confusion conceptuelle comme l'identification de la vérité à l'opinion, ce qui les conduit par exemple à affirmer qu'il y aurait des vérités fausses (par opposition aux vérités vraies). En philosophie, cette confusion est liée à une compréhension fautive du fameux « plan dialectique » compris comme oui / non / peut-être. Ramené à sa plus simple expression, le plan se résume à : "dans une première partie, je vais démontrer que oui et dans une deuxième partie, je vais démontrer que non. » Pour ces élèves, il ne semble pas aller de soi que si l'on a démontré que la réponse était " oui », alors ce ne pouvait être "non ». Certains élèves se réfugient dans la doxographie arguant qu'ils ont exposé «le pour et le contre». Il peut être bénéfique de leur rappeler qu'il s'agit de penser et non de réciter des opinions, faute de quoi ils ne sont plus les auteurs de leurs propos mais seulement les porte-voix d'autrui. Cette confusion entre opinion et 
vérité amène les élèves à adopter le relativisme le plus plat. Et dans le même temps, on observe que lorsque quelque chose les choque, les mêmes élèves se réfugient derrière l'argument d'autorité. Ainsi, par exemple, en terminale scientifique, pour illustrer les deux sources de l'erreur que sont la précipitation et la prévention, il est fréquent que j'utilise ce résultat mathématique selon lequel $0,99999 \ldots=1$. Ce résultat a priori surprenant n'est cependant pas difficile à établir. ${ }^{3}$ Or après que j'en ai proposé la démonstration au tableau, il n'est pas rare que les élèves continuent à faire preuve d'incrédulité et affirment qu'ils vont aller demander confirmation au professeur de mathématiques, privilégiant la parole d'une autorité à la force d'une démonstration (ce qui contrevient à la démarche scientifique).

On l'a vu, l'un des éléments de la contestation de la parole enseignante est la religion. En 2004, le rapport Obin sur Les signes et manifestations d'appartenance religieuse dans les établissements scolaires faisait état d'une crispation identitaire et s'inquiétait de "la montée en puissance du phénomène religieux dans les quartiers, notamment chez les jeunes ». Selon le rapport, il s'agissait de " la partie scolairement visible d'une dynamique plus vaste, souvent récente, parfois brutale. » Néanmoins, ce rapport analysant l'attitude de jeunes qui se forgeaient une identité de substitution ne concernait qu'un petit échantillon non représentatif d'établissements. Si cette évolution est "inquiétante », elle demeure malgré tout limitée. Ainsi par exemple a-t-on fait état de quelques cas seulement de refus d'observer une minute de silence après les attentats de 2015. Selon le ministère de l'éducation, environ 200 incidents de ce genre ont affecté les quelque 64000 établissements scolaires du territoire.

On peut également se demander si cette contestation n'est pas liée à la féminisation de l'enseignement ( $83 \%$ des enseignants du premier degré du secteur public, $52 \%$ des enseignants du deuxième degré du secteur public, $91 \%$ des enseignants du premier degré du secteur privé et $25 \%$ des enseignants du deuxième degré du secteur privé sont des femmes), dans la mesure où certains élèves, chez qui sont enracinées des conceptions archaïques, peuvent rechigner à obéir à des femmes.

Face à de tels propos, la situation du professeur de philosophie apparaît singulière à plusieurs titres. En tant que professeur, il est une autorité qui prétend enseigner et donc détenir un savoir mais comme philosophe, il est désireux d'atteindre une sagesse qu'il admet ne pas posséder. De plus, il semble pris dans une contradiction : apprendre à remettre en cause, n'est-ce pas aussi apprendre à remettre en cause sa propre parole ? On oscille entre l'apprentissage d'un savoir et l'enseignement d'une attitude : le geste critique. Ce dernier n'est pas propre à la philosophie, au sens où tous les enseignements visent à développer l'esprit critique, mais il est peut-être plus marqué chez les héritiers de Socrate qui

3. Soit $\mathrm{a}=0,99999 .$. Puisque multiplier par 10 consiste à décaler la virgule d'un rang vers la droite, il est aisé de voir que $10 a=9+a$. En soustrayant a de chaque côté de l'égalité, on obtient $9 a=9$. Par suite, $a=1$. 
affirmait ne savoir qu'une chose, c'est qu'il ne savait rien. Enfin, l'adolescence étant un âge où l'on cherche à sécuriser ses connaissances, comme la philosophie consiste pour une large part justement à les mettre en cause, celle-ci peut occasionner un rejet radical.

La contestation tient aussi à ce que la confiance semble rompue. Cela est d'autant plus problématique que l'école n'est pas exempte de reproches : les enquêtes Pisa révèlent de très fortes inégalités, dans le système éducatif français, entre les enfants des classes favorisées et ceux des classes défavorisées mais aussi entre enfants immigrés et non immigrés. Ces inégalités remettent en cause la méritocratie, puisque la notion de méritocratie ne signifie évidemment pas que ceux qui sont méritants doivent réussir mais que ceux qui sont également méritants doivent également réussir.

En outre, l'école n'est souvent plus perçue comme une chance mais comme une contrainte. Ainsi dans une très bonne classe de terminale $S^{4}$ à laquelle avait été soumis le sujet "Le but de l'éducation est-il de supprimer le naturel ?", sur un effectif de 34 élèves, 26 développent l'idée que l'école n'est plus un lieu de formation mais de formatage et qu'elle constitue un moule qui nie la personnalité des individus en les uniformisant ; 7 copies vont jusqu'à comparer l'école avec les Jeunesses hitlériennes. ${ }^{5}$ Les propos de ce genre sont encore plus fréquents dans d'autres sections comme les séries économiques et sociales (ES) et technologiques. La méfiance est ici liée à une conception de la liberté comme « expression de soi » qui ne peut admettre d'entrave.

À cela viennent s'ajouter des éléments parfois très prosaïques : ainsi, l'argent est une valeur reine et le revenu un gage de réussite. Réciproquement, l'estime décroît avec le revenu. Or, selon l'OCDE, les enseignants français sont parmi les plus mal payés d'Europe. Il n'est pas rare d'entendre des élèves tenir des propos selon lesquels l'enseignant est un médiocre. Ainsi une collègue de sciences économiques et sociales s'est-elle entendu dire que si elle s'y connaissait en économie, elle serait chef d'entreprise. Ce discours est loin d'être marginal.

Il y a aussi une entreprise idéologique de délégitimation venant de certains milieux. D'après un ancien président de la République, alors en exercice :

l'instituteur ne pourra jamais remplacer le curé ou le pasteur dans la transmission des valeurs et dans l'apprentissage de la différence entre le bien et le mal. ${ }^{6}$

Selon un sondage de 2014, deux tiers des Français accordent leur confiance aux enseignants, pourcentage en recul par rapport aux trois quarts qu'ils étaient deux ans avant, ce qui place les enseignants derrière les pompiers, les artisans, les bouchers ou les chefs de petites et moyennes entreprises, la confiance étant la moins grande chez les 18-24 ans.

4. Très bonne par les résultats (une dizaine d'élèves obtiendront la mention bien ou très bien au bac) mais aussi par l'attitude en classe ou plus généralement face au travail.

5. Il n'est pas rare de retrouver des propos similaires chez des étudiants en $1^{\text {re }}$ année de licence de philosophie.

6. Discours de Nicolas Sarkozy au Palais du Latran le 20 décembre 2007. 
Malgré la défiance vis-à-vis des journalistes (qui ne recueillent que $37 \%$ de confiance, selon le même sondage), l'exposition médiatique dont l'obscur enseignant ne peut se prévaloir agit comme un gage de crédibilité auprès des élèves. En témoigne leur grande confusion quand il s'agit d'art : ceux-ci identifient fréquemment " grand artiste » et « artiste connu».

Ces contestations relèvent aussi de la simple affirmation de soi contre ce qui est perçu comme l'autorité, et notamment chez les enfants et adolescents contre le monde adulte. En France, il semble que cette contestation se traduise par l'indiscipline. ${ }^{7}$ Selon Le Vallois et Aulenbacher (2006) l'adolescence est aussi l'âge de la confrontation avec les limites (sports extrêmes). Dès lors, faut-il s'étonner de la tenue de propos extrêmes? Même dans la contestation religieuse, il y a avant tout, selon le rapport Obin (2004), une affirmation identitaire. Cette contestation est ainsi parfois la manifestation d'une volonté d'expression de soi, une revendication de liberté contre la science conçue comme oppressive, au sens où on ne vote pas pour un théorème. Ainsi une enseignante s'est-elle entendu répondre par un élève qui contestait que l'homme fût allé sur la Lune : "J'ai envie de croire ce que je veux. " La contestation repose ici sur une conception naïve de la liberté comprise comme absence de toute contrainte. Il est bon de rappeler aux élèves qu'il y a contrainte et contrainte. La contrainte exercée par le brigand qui me menace d'une arme, celle du parent qui commande à son enfant d'aller à l'école et celle du sportif qui s'impose un entraînement ne sont pas du même ordre. La première est subie contre l'intérêt de celui qui la subit, la deuxième est subie dans son intérêt, la troisième est choisie.

\section{LA PROXIMITÉ ENTRE RELATIVISME ET DOGMATISME}

On a évoqué précédemment la coexistence, chez les élèves, du relativisme et du dogmatisme. Ce relativisme conduit à faire de la science une opinion comme une autre et à mettre au même niveau théorie scientifique et dogme religieux. Cependant, ce relativisme repose pour partie sur un certain nombre de considérations exactes. Les créationnistes proclament que le darwinisme n'est qu'une théorie. C'est vrai. Il est donc maladroit de leur opposer, comme on le voit faire à certains, qu'il s'agit d'un acquis définitif. Notons d'abord que de tels arguments ne sont pas nouveaux. Ils avaient été avancés dans la lettre encyclique du pape Léon XIII Providentissimus Deus ${ }^{8}$, parue peu de temps après L'origine des espèces et qui contient un véritable manuel de lutte contre le rationalisme. En plus des condamnations morales portant sur une science arrogante et sans chaleur, il proposait, en reprenant saint Augustin, d'opposer au physicien que celui-ci ne dispose que d'hypothèses incertaines qui pourront être réfutées

7. Cf. l'enquête Pisa de 2015.

8. Voir : [https://goo.gl/wtLTYa] 
ultérieurement. La science s'est déjà contredite ; elle n'est donc pas certaine. Cette mise en cause de la valeur de la science se fait en alléguant son statut hypothético-déductif et en faisant valoir l'histoire des sciences. On trouve de même, dans l'encyclique Fides et ratio ${ }^{9}$ de Jean-Paul II, une mise en cause des capacités de la raison scientifique. Cette dernière ne peut faire l'économie de la confiance - ce qui relativise sa certitude. Puisque l'on ne peut pas refaire toutes les expériences ni toutes les démonstrations, il faut donc croire ses prédécesseurs. Bref la science repose sur un acte de foi. Ces considérations sont en partie exactes. Il y a des raisons de remettre en cause les théories. Par conséquent, il n'est pas pertinent de qualifier le relativisme d'irrationnel et d'affirmer que les théories sont indiscutables. Cela serait d'autant plus maladroit que c'est précisément de ce dogmatisme que se nourrit le relativisme, tout comme le scientisme du $\mathrm{XIX}^{\mathrm{e}}$ siècle a généré le mouvement anti-science du $\mathrm{XX}^{\mathrm{e}}$ siècle. L'épistémologue Paul Feyerabend (1996) qualifie le relativisme et le dogmatisme de «jumeaux pleins de hargne »: ils s'appuient sur les mêmes postulats pour partir dans des directions opposées. Jean-Paul Jouary expose comment dogmatisme et relativisme sont liés :

Apprendre aux élèves quels ont été les résultats des sciences, sans faire intérioriser la nature des obstacles qu'il a fallu vaincre pour y parvenir, les modes de pensée que cela a supposé, le cheminement conceptuel qui y a conduit - ce n'est pas enseigner les sciences, mais faire répéter leurs conclusions comme s'il s'agissait d'opinions. Dès lors, qu'on ne s'étonne pas du voisinage de la conception dogmatique de la vérité avec le relativisme le plus plat, et de la coexistence des diplômes scientifiques avec les croyances les plus archaïques. (op. cit.)

Présenter une théorie actuelle comme une vérité indépassable, c'est précisément confondre théorie et dogme. En accréditant l'idée que ce qui est faux doit être absurde, on encourage les élèves à effectuer cette confusion. Or tel n'est pas le cas : les théories aujourd'hui abandonnées avaient des éléments en leur faveur, le géocentrisme mobilisait des arguments scientifiques, comme l'absence étonnante de force centrifuge sur une planète censée tourner à $100000 \mathrm{~km} / \mathrm{h}$ autour du soleil. Il faudra le génie de Galilée pour expliquer cette absence. Le physicien et épistémologue Pierre Duhem rappelle que de nombreux scientifiques (à commencer par Newton) adoptent le principe positiviste de l'équivalence des hypothèses : le scientifique ne prétend pas dire quelle est la réalité mais propose simplement des modèles qui en rendent efficacement compte.

Marianne Doury (1997) a montré l'incapacité d'un discours dogmatique à convaincre les tenants des parasciences. Les Lumières considéraient que la propagation du savoir amènerait à une libération. Si cet idéal n'a pas perdu sa valeur, il doit néanmoins être précisé. On ne peut se contenter de présenter un simple corpus de résultats. Georges Charpak et Henri Broch (2002) ont montré

9. Voir : [https://goo.gl/SmybEp] 
que la croyance dans les parasciences augmente globalement avec le niveau d'éducation. Selon un sondage effectué par ces auteurs, les étudiants de premier cycle scientifique accordaient plus de crédit à la torsion des cuillères d'Uri Geller qu'au phénomène relativiste de dilatation du temps. D’après ces auteurs, la culture scientifique permettrait, non de diminuer la croyance dans les parasciences, mais de donner à ces croyances une forme plus rationnelle.

\section{LA NÉCESSAIRE DISTINCTION ENTRE RATIONNEL ET RAISONNABLE}

S’agit-il alors de permettre aux élèves d'acquérir non pas tant un contenu qu'une attitude? Là encore, il y a un risque qu'on pourrait qualifier d'hypercriticisme. Si la science et le journalisme procèdent du doute, il existe cependant un moment où l'on doit y mettre fin pour considérer que l'on a atteint une certitude, c'est-à-dire que l'on estime avoir levé tout doute raisonnable. Il faut distinguer entre raisonnable et rationnel : si mille témoignages vont dans le même sens, il n'est pas irrationnel de continuer à douter - car après tout, ces mille personnes peuvent mentir ou se tromper - mais cela devient déraisonnable. De même, il existe deux formes de doute : celui qui s'oppose à la naïveté, qui est adhésion irréfléchie, et celui qui s'oppose à la confiance, qui est adhésion réfléchie. Le paradoxe est que la méfiance déraisonnable se traduit par une grande naïveté. Les élèves qui remettent en question la rotondité de la terre adhèrent sans recul à tel écrit religieux. Bref, l'esprit critique doit être exercé avec méthode, pour ne pas conduire à la crédulité. La difficulté est qu'il n'existe pas de procédure automatique pour déterminer quand le doute devient déraisonnable.

La science fait appel au raisonnable. Le géocentrisme n'est pas irrationnel mais déraisonnable tant il faut d'hypothèses ad hoc pour y souscrire. Il est important d'amener les élèves à distinguer les deux. On peut en les interrogeant les amener à admettre qu'une théorie du complot n'est pas irrationnelle mais déraisonnable. Il est préférable en effet de poser des questions afin que la réponse vienne d'eux plutôt que de l'affirmer ex cathedra. Ainsi, si l'on prend les attentats du 11 septembre, sans doute n'est-il pas irrationnel de considérer que ceux-ci ont été orchestrés par le gouvernement américain mais cela est-il raisonnable ? Le risque d'être découvert et les conséquences sont trop importants pour que cela soit probable. À l'inverse, détournement et attentat suicide sont plus courants et donc plus vraisemblables. Tel était déjà l'argumentaire de Hume (1983) contre les miracles : si quelqu'un dit avoir vu un miracle, il y a trois possibilités : a) il a vu un miracle ; b) il ment ; c) il se trompe. Un miracle étant par définition une violation des lois de la nature, il est infiniment plus raisonnable de croire que je suis face à une chose qui se produit souvent, à savoir l'erreur ou la tromperie. Bref, il s'agit de faire entrer les élèves dans un processus d'argumentation et notamment de les faire réfléchir à la solidité des affirmations. 
En plus des idéologies qui propagent des contre-vérités, les propos extrêmes des élèves relèvent de multiples confusions : sur la liberté, la science, la rationalité, etc. C'est pourquoi il importe de lever ces confusions, d'instruire les élèves de ce qu'est la critique : montrer la nature de cette méfiance qui ne dégénère pas en hypercriticisme. Et c'est pourquoi, cette méfiance n'est pas antagonique avec la confiance, confiance qu'il s'agit de restaurer en rappelant que loin de supprimer la liberté, l'éducation la réalise, que le fait d'apprendre les mêmes choses ne fait pas de nous les mêmes individus : en apprenant les mathématiques, l'un deviendra scientifique, un autre apprendra à ne pas se faire « rouler» quand on lui rend la monnaie. Elle augmente donc le choix et supprime la dépendance. Il serait aussi bon de renforcer l'éducation aux médias et à l'information. De même, il n'est pas inutile de rappeler que le mot « chercher » possède deux significations. L'une renvoie à la consultation des travaux d'autrui, l'autre à la production personnelle de connaissances nouvelles. Si Internet facilite la première conception, il faut promouvoir la seconde, surtout en un âge où l'on est soucieux de s'affirmer. Enfin, on ne saurait trop insister sur la nécessité d'accentuer la dimension historique et philosophique des sciences. Trop d'enseignants estiment encore qu'il ne s'agit là que de superfluités. Or les discours des élèves viennent de conceptions épistémologiques naïves. Voir en la science un simple corpus de résultats encourage à une fétichisation sans esprit critique qui se paie par une méfiance et un rejet. Une théorie n'est pas une vérité absolue ; elle n'est pas pour autant une opinion comme les autres : une théorie qui articule bon nombre de faits d'expériences est plus crédible qu'un dogme qui s'en passe. Une meilleure formation des enseignants serait sans doute nécessaire. La « mission Dominique Lecourt », commandée par Claude Allègre, a conclu à la nécessité de donner des cours de philosophie des sciences aux étudiants (et futurs enseignants) de science, cours qui leur faisait défaut. Concluons : il ne s'agit pas tant d'enseigner la vérité que, pour reprendre les mots de Jean-Paul Jouary, d'« enseigner les exigences passées et présentes de sa recherche, les façons passées et présentes d'y répondre, les résultats obtenus hier et aujourd'hui » (op. cit.). Si cette manière de faire peut paraître plus relativiste que l'enseignement dogmatique d'une vérité, elle est moins susceptible d'y mener : il n'y a rien de pire qu'un amoureux déçu.

\section{BibLIOGRAPHIE}

BRONNER G. (2013) : La démocratie des crédules, Paris : Presses Universitaires de France.

CHARFI F. F. (2013) : La science voilée, Paris : Odile Jacob.

CHARPAK G., BROCH H. (2002) : Devenez sorciers, devenez savants, Paris: Odile Jacob.

DOURY M. (1997) : Le débat immobile, Paris : éditions Kimé. 
DUBESSY J., LECOINTRE G. (dir.) (2001) : Intrusions spiritualistes et impostures intellectuelles en sciences, Paris : Syllepse.

FEYERABEND P (1996): Dialogues sur la connaissance, Paris : Seuil.

HUME D. (1983) : Enquête sur l'entendement humain, Paris : Flammarion.

JOUARY J.-P. (2002) : Enseigner la vérité? Essai sur les sciences et leurs représentations, Paris : L'Harmattan.

LECOURT D. (1992) : L'Amérique entre la Bible et Darwin, Paris : Presses Universitaires de France.

LECOURT D. (1999): L'enseignement de la philosophie des sciences. Rapport au ministre de l'éducation nationale, de la recherche et de la technologie. [https://goo.gl/ jzdGLG]

LE VALlOIS P., AULENBACHER A. (2006) : Les ados et leurs croyances, Paris : Les éditions de l'atelier.

OBIN J.-P. (2004): Les signes et manifestations d'appartenance religieuse dans les établissements scolaires Rapport à monsieur le ministre de l'éducation nationale, de l'enseignement supérieur et de la recherche, juin, $\mathrm{n}^{\circ} 20$. [https://goo.gl/rA7k9N]

WEBER M. (1963) : Le savant et le politique, Paris : 10/18. 
